\title{
Abortion and mental health disorders: evidence from a 30-year longitudinal study ${ }^{\dagger}$
}

David M. Fergusson, L. John Horwood and Joseph M. Boden

\section{Background}

Research on the links between abortion and mental health has been limited by design problems and relatively weak evidence.

\section{Aims}

To examine the links between pregnancy outcomes and mental health outcomes.

\section{Method}

Data were gathered on the pregnancy and mental health history of a birth cohort of over 500 women studied to the age of 30 .

\section{Results}

After adjustment for confounding, abortion was associated with a small increase in the risk of mental disorders; women who had had abortions had rates of mental disorder that were about 30\% higher. There were no consistent associations between other pregnancy outcomes and mental health. Estimates of attributable risk indicated that exposure to abortion accounted for $1.5 \%$ to $5.5 \%$ of the overall rate of mental disorders.

\section{Conclusions}

The evidence is consistent with the view that abortion may be associated with a small increase in risk of mental disorders. Other pregnancy outcomes were not related to increased risk of mental health problems.

\section{Declaration of interest}

None. Funding detailed in Acknowledgements.
Over the past two decades there has been increasing research into mental health outcomes associated with induced abortion. These outcomes include depression, ${ }^{1-10}$ anxiety, ${ }^{6,8,11,12}$ substance use, ${ }^{8,13}$ and suicidal behaviour and self-harm. ${ }^{8,14}$ The findings from this research have been both controversial and inconsistent, with a number of studies reporting adverse effects associated with abortion $^{3,6,8,10,13}$ but others finding no association. ${ }^{1,2,4,5,12}$ Research in this area has faced a number of complications that have prevented clear conclusions about whether or not exposure to induced abortion is associated with increased (or decreased) risks of mental health problems in women having unwanted pregnancies. First, studies in this area have been subject to a number of threats to validity that include inadequate ascertainment of exposure to abortion, limited assessment of mental health outcomes and failure to control confounding. ${ }^{8,15-18}$ These threats to validity are pervasive and no existing study has been able to overcome all of them. ${ }^{15}$ These technical problems of research design have been exacerbated by the fact that the issue of mental health effects of abortion has been hotly contested by pro-life and pro-choice advocates, resulting in a situation in which weak research evidence has been used to support strongly stated opinions on the harms or benefits associated with abortion. ${ }^{15,16}$ Finally, there has been an unfortunate tendency in the literature on this topic for study findings to coincide with the ideological views of authors about the desirability of abortion. ${ }^{15,19}$ This combination of limited evidence, controversy and strongly stated opinion has made research into the mental health effects of abortion a contested area and there has been wide variation in scientific views of the matter. ${ }^{3,20-22}$

In this paper we report an analysis of data from a 30 -year longitudinal study in which we seek to examine the extent to which variations in pregnancy outcomes, including induced abortion, live birth and pregnancy loss, are associated with increased (or decreased) risks of a range of common mental health problems (major depression, anxiety disorders, suicidal ideation,

†See invited commentaries, pp. 452-454 this issue. alcohol dependence and illicit drug dependence). This analysis is an extension of an earlier study ${ }^{8}$ that examined the links between abortion and mental health in the same birth cohort.

\section{Method}

The data used in this analysis were gathered over the course of the Christchurch Health and Development Study (CHDS). The CHDS is a longitudinal study of a birth cohort of 1265 children born in the Christchurch urban region in New Zealand who have been studied at birth, 4 months, 1 year and annual intervals to age 16 years, and again at ages 18,21, 25 and 30 . The present analysis is based on the cohort of 534 women for whom information on pregnancy history and mental health outcomes was available. However, since not all women were assessed on all occasions, the sample sizes used in the analysis range between 507 and 524, depending on the timing of assessment of pregnancy history and mental health. These samples represent between $80 \%$ and $83 \%$ of the original cohort of 630 women. All data were collected only on the basis of signed consent from participants. The study had ethical approval from the Canterbury ethics committee.

\section{Pregnancy and abortion (15-30 years)}

At each assessment from age 15 to 30 years participants were questioned about any pregnancies occurring since the previous assessment, and the timing and outcome of each reported pregnancy was recorded. Participants were also questioned about their reaction to each pregnancy and the extent to which this had caused them to be upset or distressed. At age 30, as a check on the accuracy of the prospectively gathered pregnancy data, all participants were asked to provide a summary of their full pregnancy history. As well as information on timing and outcome, participants were also questioned about whether the pregnancy was wanted or unwanted, and their initial reaction to the pregnancy at the time. Initial reactions were coded on a 5-point scale from very happy to very unhappy/distressed. Comparison 
of the prospective and retrospective reports of pregnancy history showed that for just under $90 \%$ of women the two reports were in agreement.

For the purposes of the present analysis the measures of pregnancy history were defined using a combination of both prospective and retrospective reports. Using the information on timing and outcome for each reported pregnancy, the woman's pregnancy history for any given period of interest was classified using four dichotomous measures of pregnancy outcomes.

(a) Whether the woman reported having an elective abortion during the interval.

(b) Whether the women experienced a pregnancy loss during the interval. Pregnancy loss was defined to include miscarriage, stillbirth and termination of ectopic pregnancy.

(c) Whether the woman had a live birth during the interval for which she reported an adverse reaction to the pregnancy. An adverse reaction was defined to include where the woman reported that the pregnancy was unwanted or that she had been distressed or very distressed on learning of the pregnancy.

(d) Whether the woman had a live birth during the interval with no adverse reaction to the pregnancy.

Based on the combined report data, 284 women (53\% of the cohort) reported a total of 686 pregnancies before age 30 . These pregnancies included a total of 153 abortions (occurring to 117 women), 138 pregnancy losses $(n=95), 66$ live births $(n=52)$ that resulted from an unwanted pregnancy or one that provoked an adverse reaction, and 329 live births $(n=197)$ resulting from a pregnancy for which there was no reported adverse reaction.

\section{Mental health (15-30 years)}

At each assessment from age 16 to 30 years, participants were questioned about mental health issues since the previous assessment using structured questionnaires based on the Diagnostic Interview Schedule for Children (DISC) $)^{23}$ at age 16 years and the Composite International Diagnostic Interview ${ }^{24}$ at ages 1830 years, supplemented by additional measures. From this questioning it was possible to ascertain the proportion of young women who met DSM-IV ${ }^{25}$ diagnostic criteria for the following disorders during the intervals $15-18,18-21,21-25$ and $25-30$ years: major depression, anxiety disorders (including generalised anxiety, panic disorder, agoraphobia, social phobia and specific phobia), alcohol dependence and illicit drug dependence. In addition, measures of DSM-IV disorders were supplemented by measures of self-reported suicidal ideation. Finally, to provide an overall measure of the burden of mental disorder, the individual measures were summed to obtain a count of the number of mental health problems reported for each interval.

\section{Covariate factors}

Measures of childhood socio-economic circumstances

Maternal and paternal education levels were assessed at the time of the cohort member's birth using a 3-point scale (no formal qualifications, secondary qualifications, tertiary qualifications). Family socio-economic status was assessed at birth using the Elley-Irving revised index of socio-economic status for New Zealand. ${ }^{26}$ Family living standards were assessed on the basis of an interviewer rating at each interview from birth to age 10 years. Ratings were made on a 5-point scale from very good to very poor. These ratings were averaged over the 10 -year period to provide an overall measure of the family's average living standard during this period. At each assessment from age 1 to 10 years, estimates were obtained of the family's gross annual income for the past 12 months.

\section{Measures of parental adjustment/family functioning}

Using detailed information on patterns of family change gathered over the interval from birth to 15 years, a measure of family instability was constructed on the basis of a count of the number of changes of parents experienced by the child by age 15 . At age 18, participants were questioned using items from the Conflict Tactics Scale ${ }^{27}$ concerning incidents of inter-parental violence that they may have witnessed during childhood ( $<16$ years). These items were combined to provide an overall measure of the extent of inter-parental violence. ${ }^{28}$ The reliability of this scale was $\alpha=0.88$.

When participants were aged 11 years, parents were questioned about their own history of illicit drug use. When participants were aged 15 years, parents were further questioned about their history of mental health problems, problems with alcohol, and involvement in criminal offending. These reports were used to classify participants on four dichotomous measures reflecting whether any parent had a reported history of illicit drug use, mental health problems, alcohol problems or criminality respectively. The quality of parental-child attachment in adolescence was assessed at age 15 years using the Armsden \& Greenberg $^{29}$ scale of parental attachment $(\alpha=0.87)$.

\section{Measures of exposure to child abuse}

At age 18 and 21 years, participants were questioned about their experience of sexual abuse in childhood $\left(<16\right.$ years). ${ }^{30}$ Individuals were classified as having experienced childhood sexual abuse if they reported at either age 18 or 21 any episode of abuse involving physical contact with a perpetrator. Participants were also questioned at age 18 and 21 years about the extent, on a 5 -point scale, ${ }^{31}$ to which their parents used physical punishment during childhood ( $<16$ years). Individuals were classified as having experienced physical child abuse if they reported at either age 18 or 21 that at least one parent had regularly used physical punishment, had used physical punishment too often or too severely, or had treated them in a harsh and abusive manner.

\section{Measures of individual characteristics and educational} achievement

Child neuroticism was assessed at age 14 years using a short-form version of the neuroticism scale of the Eysenck Personality Inventory. ${ }^{32}$ The reliability of this scale was $\alpha=0.80$. Child self-esteem was assessed at age 15 years using the Coopersmith Self-Esteem Inventory. ${ }^{33}$ The reliability of this scale was $\alpha=0.87$. Child novelty-seeking was assessed at age 16 years using the novelty-seeking scale of the Tridimensional Personality Inventory. ${ }^{34}$ The reliability of this scale was $\alpha=0.76$. At age 7,8 and 9 years, the extent to which participants exhibited tendencies to conduct disordered and oppositional behaviours was assessed using a scale that combined items from the Rutter ${ }^{35}$ and Conners ${ }^{36,37}$ child behaviour rating scales. Separate ratings were obtained from the child's parent and class teacher. Parent and teacher ratings were summed for each year and then averaged over the interval from 7 to 9 years to provide a robust measure of the child's tendencies to conduct problems. The reliability of the resulting scale was $\alpha=0.97$. Child IQ was assessed at age 8 years using the revised Wechsler Intelligence Scale for Children. ${ }^{38}$ The split half reliability of this scale was 0.93 .

At each assessment from age 11 to 13 years, the child's class teacher was asked to rate the child's performance in each of the five areas of the curriculum (reading, handwriting, written 
expression, spelling, mathematics) using a 5-point scale ranging from $1=$ very good to $5=$ very poor. To provide a global measure of the child's educational achievement over the interval from 11 to 13 years, the teacher ratings were summed across years and curriculum areas and then averaged to provide a teacher-rating grade point average for each child. The reliability of this measure was $\alpha=0.96$.

\section{Measures of adolescent adjustment}

At age 18, participants were questioned about their sexual behaviours, including the age of first intercourse. Young women who reported that they had first had sex before age 16 were classified as having early sexual onset.

At age 15, participants were questioned about their use of tobacco, alcohol and cannabis. Tobacco use was assessed on the basis of a 5-point scale reflecting the current frequency of cigarette smoking at age 15. This scale ranged from 'non-smoker' through to 'daily smoker'. The frequency of alcohol use in the past 12 months was assessed using a 6-point scale that ranged from 'never' to 'almost every day'. In addition, a dichotomous measure of cannabis use was created based on the young person's report of use in the past 12 months.

At age 15, young people were administered a mental health interview that combined components of $\mathrm{DISC}^{23}$ and other measures to assess a range of DSM-III- $\mathrm{R}^{39}$ disorders in the cohort over the previous 12 months. This information was used to construct DSM-III-R diagnoses of major depression and anxiety disorders, including overanxious disorder, generalised anxiety disorder, social phobia and simple phobia. In addition, individuals were also questioned about the frequency of suicidal thoughts in the previous 12 months.

\section{Time-dynamic lifestyle and other factors}

At each assessment from age 18 onwards, participants were questioned about aspects of their living arrangements since the previous assessment including: (a) living with parents and age of leaving the family home; and (b) entry into cohabiting relationships. In addition, participants were questioned about other adverse life events occurring in each year since the previous assessment using a life-event checklist based on the Feeling Bad Scale. ${ }^{40}$ This information was used to classify participants on four measures reflecting the extent of exposure to employment problems, partner relationship problems, serious illness or death in the family, and sexual or physical violence victimisation within any given interval. Finally, to control for the changing history of prior mental health, a lagged measure of the number of mental health problems observed in the previous assessment period was also considered.

\section{Statistical analysis}

The associations (risk ratios) between pregnancy history and mental health at ages $15-18,18-21,21-25$ and $25-30$ years (online Table DS1) were estimated by fitting random-effects models to the repeated-measures data for each outcome and each measure of pregnancy history (abortion, pregnancy loss, live birth with adverse reaction, other live birth). For dichotomous outcomes (depression, anxiety, suicidal ideation, substance dependence) logistic regression models were fitted, whereas for the count of number of mental health problems Poisson regression was used. For each outcome $(Y)$ the general model fitted was of the form:

$$
G\left(Y_{i t}\right)=B_{0 t}+B_{1} X_{i t}+v_{1}+e_{i t}
$$

$G\left(Y_{i t}\right)$ was the log odds of $Y$ for the $i$-th individual in the $t$-th time interval for dichotomous outcomes or the log rate of problems for the $i$-th individual in the $t$-th time interval for the count of the number of mental health problems; $X_{i t}$ was a timedynamic dichotomous variable reflecting the pregnancy history outcome (abortion, pregnancy loss, live birth with adverse reaction, or other live birth) for the $i$-th individual up to the $t$-th interval; $v_{i}$ was an individual specific random effect assumed to be uncorrelated with $X_{i t}$; and $e_{i t}$ was the disturbance term for the model. The intercept $B_{0}$ was permitted to vary with time $t$ to allow for changes in the base rate of each outcome over time. In each case, an estimate of the pooled risk ratio of disorder (odds ratios for dichotomous outcomes, incidence rate ratio for the problem count) and corresponding 95\% confidence interval was obtained from the fitted coefficient $B_{1}$ and its standard error in the usual way $e^{B 1 \pm 1.96 s . e .(B 1)}$. To avoid problems associated with repeated significance testing of multiple correlated outcomes, tests of significance of the pooled associations were restricted to the models for the count of number of mental health problems.

Risk ratio estimates for each measure of pregnancy history adjusted for other pregnancy outcomes (Table 1) were obtained by extending the models in the above equation to include all four measures of pregnancy history. Thus:

$$
G\left(Y_{i t}\right)=B_{0 t}+B_{1} X_{1 i t}+B_{2} X_{2 i t}+B_{3} X_{3 i t}+B_{4} X_{4 i t}+v_{i}+e_{i t}
$$

$X_{1 i t}, X_{2 i t}, X_{3 i t}, X_{4 i t}$ were time-dynamic dichotomous measures reflecting exposure of the $i$-th participant to abortion, pregnancy loss, live birth with adverse reaction, or other live birth respectively. Two models were fitted for each mental health outcome: (a) a model in which the pregnancy history measures $\left(X_{1 i t}, X_{2 i t}\right.$, $\left.X_{3 i t}, X_{4 i t}\right)$ were assessed up to and concurrently with the observation interval for mental health outcomes; (b) a model in which the pregnancy history outcomes were assessed as lagged measures in the 5 years prior to the beginning of the interval in which the mental health outcome was assessed. The randomeffects models were then further extended to obtain risk ratio estimates adjusted for the series of fixed and time-dynamic covariate factors described previously (Table 2). In fitting the covariate adjusted models, all covariates were initially considered for inclusion in each model. The covariate set was then refined using methods of forwards and backwards variable elimination to identify a stable set of covariates that were significant in at least one of the models fitted. The final analyses were conducted using only these covariates.

All models were fitted using Stata version 8 for Windows. Estimates of the attributable fraction of mental health problems owing to abortion were derived from the adjusted rate ratio measures using the methods described by Bruzzi et al. ${ }^{41}$

\section{Sample bias}

As noted previously the sample sizes available for analysis represented $80-83 \%$ of the initial sample of 630 women who entered the study at birth. To test for selection bias arising from the processes of sample attrition, the sample of women included in the analysis was compared with the remaining cohort on a series of measures collected at the time of birth. These comparisons suggested evidence of small but statistically significant $(P<0.05)$ tendencies for women from socio-economically disadvantaged backgrounds (low parental education, low socio-economic status family, single-parent family) to be under-represented in the analysis sample. To examine the extent to which the study findings may have been influenced by these small biases, the analyses were repeated using the data weighting methods described by Carlin 


\begin{tabular}{|c|c|c|c|c|}
\hline \multirow[b]{2}{*}{ Measure } & \multirow[b]{2}{*}{$\begin{array}{c}\text { Abortion } \\
\text { RR (95\% Cl) }\end{array}$} & \multirow[b]{2}{*}{$\begin{array}{l}\text { Pregnancy loss } \\
\text { RR }(95 \% \mathrm{Cl})\end{array}$} & \multicolumn{2}{|c|}{ Live birth, RR $(95 \% \mathrm{Cl})$} \\
\hline & & & $\begin{array}{l}\text { Unwanted/adverse } \\
\text { reaction }\end{array}$ & $\begin{array}{l}\text { No adverse } \\
\text { reaction }\end{array}$ \\
\hline \multicolumn{5}{|l|}{ Concurrent models } \\
\hline Major depression & $2.04(1.36-3.07)$ & $1.99(1.21-3.28)$ & $1.97(1.11-3.48)$ & $0.69(0.46-1.04)$ \\
\hline Anxiety disorder & $2.10(1.38-3.19)$ & $2.12(1.28-3.49)$ & $1.46(0.80-2.66)$ & $1.05(0.70-1.58)$ \\
\hline Suicidal ideation & $2.07(1.19-3.57)$ & $3.08(1.62-5.86)$ & $1.37(0.63-3.00)$ & $0.99(0.57-1.73)$ \\
\hline Alcohol dependence & $1.89(0.86-4.17)$ & $3.30(1.33-8.21)$ & $0.44(0.10-1.83)$ & $0.74(0.31-1.81)$ \\
\hline Illicit drug dependence & $6.64(2.83-15.61)$ & $1.68(0.55-5.14)$ & $1.36(0.40-4.63)$ & $0.53(0.19-1.46)$ \\
\hline Number of mental health problems & $1.49(1.24-1.80)$ & $1.48(1.19-1.83)$ & $1.18(0.91-1.53)$ & $0.91(0.75-1.09)$ \\
\hline \multicolumn{5}{|l|}{ 5-year lagged models } \\
\hline Major depression & $1.54(0.92-2.59)$ & $1.29(0.67-2.50)$ & $1.72(0.82-3.61)$ & $1.51(0.90-2.52)$ \\
\hline Anxiety disorder & $2.72(1.59-4.66)$ & $2.45(1.23-4.91)$ & $1.17(0.51-2.67)$ & $1.75(1.02-3.00)$ \\
\hline Suicidal ideation & $2.26(1.17-4.38)$ & $2.15(0.96-4.83)$ & $1.46(0.52-4.09)$ & $2.42(1.24-4.71)$ \\
\hline Alcohol dependence & $5.33(1.90-14.97)$ & $2.29(0.62-8.39)$ & $0.37(0.03-4.12)$ & $1.15(0.34-3.87)$ \\
\hline Illicit drug dependence & $4.82(1.91-12.18)$ & $3.85(1.05-14.09)$ & $0.37(0.05-2.80)$ & $0.36(0.08-1.58)$ \\
\hline Number of mental health problems & $1.48(1.18-1.85)$ & $1.26(0.96-1.67)$ & $1.05(0.74-1.49)$ & $1.27(1.00-1.61)$ \\
\hline
\end{tabular}

\begin{tabular}{|c|c|c|c|c|c|}
\hline \multirow[b]{2}{*}{ Measure } & \multirow[b]{2}{*}{$\begin{array}{c}\text { Abortion } \\
\text { RR }(95 \% \mathrm{Cl})\end{array}$} & \multirow[b]{2}{*}{$\begin{array}{c}\text { Pregnancy loss } \\
\text { RR }(95 \% \mathrm{CI})\end{array}$} & \multicolumn{2}{|c|}{ Live birth, RR $(95 \% \mathrm{Cl})$} & \multirow[b]{2}{*}{$\begin{array}{l}\text { Significant } \\
\text { covariates }^{\text {a }}\end{array}$} \\
\hline & & & $\begin{array}{l}\text { Unwanted/ } \\
\text { adverse reaction }\end{array}$ & $\begin{array}{l}\text { No adverse } \\
\text { reaction }\end{array}$ & \\
\hline \multicolumn{6}{|l|}{ Concurrent models } \\
\hline Major depression & $1.58(1.08-2.32)$ & $1.14(0.70-1.85)$ & $1.60(0.94-2.72)$ & $0.71(0.47-1.05)$ & $3-5,7,12-17$ \\
\hline Anxiety disorder & $1.55(1.03-2.32)$ & $1.41(0.86-2.32)$ & $1.22(0.69-2.15)$ & $1.12(0.75-1.68)$ & $5,8,14-17$ \\
\hline Suicidal ideation & $1.35(0.77-2.38)$ & $1.96(1.00-3.83)$ & $0.93(0.41-2.13)$ & $1.02(0.57-1.83)$ & $3-5,9,12,14-16$ \\
\hline Alcohol dependence & $1.19(0.56-2.55)$ & $2.23(0.92-5.41)$ & $0.48(0.12-1.89)$ & $0.68(0.28-1.64)$ & $4,12-14,17$ \\
\hline Illicit drug dependence & $3.56(1.59-7.98)$ & $0.87(0.29-2.56)$ & $1.12(0.35-3.63)$ & $0.58(0.22-1.50)$ & $2,3,10,11,14,16,17$ \\
\hline Number of mental health problems & $1.37(1.16-1.62)$ & $1.25(1.01-1.53)$ & $1.11(0.88-1.41)$ & $0.93(0.78-1.11)$ & $3-7,12,14-17$ \\
\hline \multicolumn{6}{|l|}{ 5-year lagged models } \\
\hline Major depression & $1.31(0.77-2.22)$ & $0.76(0.39-1.50)$ & $1.66(0.80-3.45)$ & $1.15(0.68-1.96)$ & $3-5,7,15,16$ \\
\hline Anxiety disorder & $2.13(1.24-3.64)$ & $2.10(1.05-4.20)$ & $1.17(0.51-2.65)$ & $1.44(0.84-2.46)$ & $5,6,15$ \\
\hline Suicidal ideation & $1.61(0.82-3.18)$ & $1.47(0.63-3.39)$ & $1.02(0.35-3.00)$ & $1.85(0.94-3.65)$ & $3,4,9$ \\
\hline Alcohol dependence & $2.88(1.01-8.18)$ & $1.33(0.36-4.96)$ & $0.41(0.04-4.28)$ & $1.19(0.36-4.00)$ & $1,4,9,16$ \\
\hline Illicit drug dependence & $2.85(1.09-7.44)$ & $1.30(0.33-5.19)$ & $0.22(0.02-2.77)$ & $0.30(0.07-1.35)$ & $2,3,7,10$ \\
\hline Number of mental health problems & $1.32(1.05-1.67)$ & $1.06(0.79-1.43)$ & $1.05(0.73-1.50)$ & $1.22(0.96-1.55)$ & $3-7,15,16$ \\
\hline \multicolumn{6}{|c|}{ 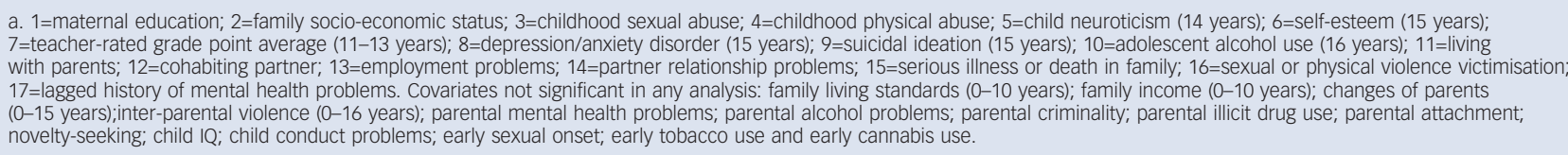 } \\
\hline
\end{tabular}

et al. ${ }^{42}$ These analyses produced almost identical conclusions to the results reported here, suggesting that the findings were unlikely to have been influenced by selection bias.

\section{Results}

\section{Associations between pregnancy outcomes and mental health (15-30 years)}

Online Table DS1 shows the relationships between a series of mental health outcomes during the intervals 15-18, 18-21, 2125 and 25-30 years and the women's pregnancy history to ages $18,21,25$ and 30 years respectively. Mental health outcomes include measures of major depression, anxiety disorder, suicidal ideation, alcohol dependence, illicit drug dependence and a count of the total number of mental health problems. Pregnancy history is summarised by four dichotomous measures representing whether the women had experienced any of the following outcomes by a given age: an abortion; a pregnancy loss (miscarriage, stillbirth, ectopic pregnancy); a live birth from a pregnancy that either was unwanted or caused the woman to have an adverse reaction to becoming pregnant; and a live birth with no adverse reaction to the pregnancy. Online Table DS1 reports associations (risk ratios (RRs)) between each measure of pregnancy history and each mental health outcome (see Method). For the measure of total number of mental health problems the RR is estimated by the incidence rate ratio (IRR), and for other outcomes by the odds ratio (OR). Online Table DS1 leads to the following conclusions.

(a) Exposure to induced abortion was consistently associated with increased rates of mental disorders, with ORs for individual disorders ranging from 1.86 to 7.08 . These trends are reflected in the fact that those exposed to abortion between ages 15-30 had overall rates of mental health problems that were 1.54 (95\% CI $1.28-1.85)$ times higher than those not exposed to abortion $(P<0.001)$.

(b) Exposure to pregnancy loss was also associated with modest but consistent increases in risks of mental health problems, with ORs for individual disorders ranging from 1.76 to 3.30 . These trends are reflected in the fact that, overall, those who had experienced pregnancy loss had rates of mental disorder 
that were 1.49 (95\% CI 1.21-1.84) times higher than those who had not $(P<0.001)$.

(c) Having a live birth that was associated with an unwanted/ adverse reaction to pregnancy was associated with modest increases in risks of all individual outcomes except for alcohol dependence. For alcohol dependence the OR was 0.54 , and for other disorders it ranged from 1.77 to 2.25 . The overall rate of disorder for those who reported an unwanted/adverse reaction was 1.31 (95\% CI 1.01-1.69) times higher than for those who did not $(P<0.05)$.

(d) Associations between mental health outcomes and having a live birth with no reported adverse reaction were generally weak and inconsistent, with ORs ranging from 0.72 to 1.41 . This trend is reflected in an overall rate of mental disorder that was only 1.03 (95\% CI $0.86-1.22)$ times higher than for those who had not experienced a live birth with no adverse reaction $(P>0.75)$.

\section{Adjustment for associations between pregnancy outcomes}

The results in online Table DS1 do not take account of the interrelationships between various pregnancy outcomes, reporting just on the contemporaneous relationships between pregnancy outcomes and mental health. These issues are addressed in Table 1, which shows estimates of the RRs between rates of mental health problems and each pregnancy outcome adjusted for other pregnancy outcomes. These estimates were obtained by fitting the random-effects model described in the Method. The associations are reported for two different models: (a) a model in which pregnancy outcomes were assessed concurrently with mental health (as in online Table DS1); and (b) a lagged model in which exposure to the pregnancy outcome occurred in the 5 years prior to the interval in which mental health was assessed. Table 1 leads to the following general conclusions.

\section{Concurrent model}

In all cases, RR estimates for exposure to abortion and exposure to pregnancy loss were greater than 1 , implying that exposure to these conditions was associated with increased risks of concurrent mental health problems. Overall, those exposed to induced abortion had rates of mental health problems that were 1.49 (95\% CI 1.24-1.80) times higher than the rates for those who did not become pregnant $(P<0.001)$, whereas exposure to pregnancy loss was associated with a 1.48 (95\% CI $1.19-1.82)$ times increase $(P<0.001)$. Risk ratios for live birth with an unwanted/adverse reaction tended to be more modest, with an overall rate of mental health problems that was only $1.18(95 \%$ CI 0.91-1.53) times the rate for those who did not become pregnant $(P>0.20)$. In contrast, other live birth was associated with reduced risks of most disorders, with an overall RR of disorder of 0.91 (95\% CI 0.75-1.09) compared with women who did not become pregnant $(P>0.30)$.

\section{The 5-year lagged model}

The results of the 5-year lagged model were in most respects very similar to the findings from the concurrent model. Exposure to induced abortion was associated with consistently increased risks of mental health problems, with women who had had abortions having overall rates of mental health problems that were 1.48 (95\% CI 1.18-1.85) times the rates for those who had not become pregnant $(P<0.001)$. Pregnancy loss was also associated with increased risks of mental health problems, with women who had had such losses having overall rates of mental health problems that were 1.26 (95\% CI 0.96-1.67) times higher than those who had not become pregnant $(P=0.10)$. Having a live birth with an unwanted/adverse reaction was associated with a modest increase in rates of internalising disorders (depression, anxiety) and suicidal ideation, but a reduction in the risks of substance use disorders, with an overall RR of disorder of 1.05 (95\% CI 0.74-1.49) that was no higher than for those who had not become pregnant $(P>0.75)$. However, in contrast to the concurrent model, those who had had a live birth without an adverse reaction appeared to have elevated risks of subsequent disorder for most mental health outcomes (particularly for anxiety disorder and suicidal ideation). Overall, having an other live birth was associated with a $1.27(95 \%$ CI $1.00-1.61)$ times increase in rates of mental disorder $(P=0.05)$.

\section{Covariate adjusted results}

A limitation of the analysis in Table 1 is that the associations between pregnancy outcomes and mental health do not take into account potential confounding factors that might be associated with increased risks of various pregnancy outcomes and/or mental health outcomes. To address this issue the associations in Table 1 were adjusted for a series of confounding covariates (see Method). These covariates included measures of: childhood socio-economic circumstances; childhood family functioning; parental adjustment; exposure to abuse in childhood; individual characteristics; educational achievement; adolescent adjustment; and timedynamic lifestyle and related factors.

Table 2 shows the estimated covariate adjusted RR and 95\% CIs for each mental health outcome estimated by the concurrent and lagged models. After adjustment, both sets of models yielded a similar set of conclusions.

(a) For both models there was consistent evidence that even after extensive covariate adjustment, exposure to abortion was associated with a modest but detectable increase in rates of mental disorder. The concurrent data suggested that after adjustment for confounding those exposed to abortion had rates of mental health problems that were 1.37 (95\% CI 1.16-1.62) times higher than for those who had not become pregnant $(P<0.001)$. The lagged model produced a slightly lower estimate of 1.32 (95\% CI 1.05-1.67, $P<0.05$ ).

(b) Pregnancy loss was associated with a modest increase in the rate of problems using the concurrent measures of pregnancy outcome, with those who experienced a pregnancy loss having a rate of mental health problems that was 1.25 (95\% CI 1.011.53) times the rate for those who were never pregnant $(P<0.05)$. However, under the lagged model, pregnancy loss was not associated with later outcomes, with an adjusted RR of 1.06 (95\% CI $0.79-1.43, P>0.70)$.

(c) For both models, having a live birth, whether with or without an unwanted/adverse reaction, was not associated with significant increases in the overall rate of mental health problems when due allowance was made for confounding variables.

Inspection of the results in Table 2 suggests that the reasons for the higher rates of mental disorder among women exposed to induced abortion were owing to small but consistent tendencies for those exposed to abortion to be at increased risks of a range of adverse mental health outcomes, with these trends being most marked for illicit drug dependence in the concurrent model, and anxiety disorders, alcohol dependence and illicit drug dependence in the lagged model. Odds ratios for specific disorders ranged from 1.19 to 3.56 for the concurrent model and from 1.31 to 2.88 for the lagged model. 


\section{Sensitivity analysis}

The models in Tables 1 and 2 are a subset of the models that could be fitted to these data. In particular, there are two key decisions that could affect the outcomes of the modelling process. The first was the data-set used to analyse the results. As explained in the Method, pregnancy data were available from both prospective and retrospective reports and the present analysis is based on a data-set that combined both retrospective and prospective report data. A second way in which the analysis could vary was with the choice of the lag between the measures of pregnancy history and the assessment of mental health outcomes. To explore the consequences of these decisions, a sensitivity analysis was conducted by replicating the analysis of the effect of abortion on the total number of mental health problems using three different strategies to define pregnancy history (combined data, prospective data, retrospective data) and using four lag rules (concurrent, lagged 5 years, 4 years and 3 years).

The results of this analysis are shown in Table 3, which gives IRR estimates and 95\% CIs for the effects of abortion on overall rates of mental health outcomes for the different models. Table 3 shows that the results of the analyses were highly consistent:

(a) the combined-data models yielded a range of IRRs that varied from 1.37 to 1.24 with a median value of 1.34 .

(b) the prospective-data models yielded a range from 1.45 to 1.29 with a median value of 1.33 .

(c) the retrospective-data models yielded a range from 1.30 to 1.23 with a median value of 1.26 .

Among the lagged models there was a tendency for RR estimates to reduce with shorter lags, with the IRRs for the 5-year lagged model ranging from 1.34 to 1.26 compared with 1.29 to 1.23 for the 3-year lagged model.

All results are consistent with the conclusion that even following extensive covariate adjustment, exposure to induced abortion was associated with a small but consistent increase in rates of mental health problems. However, although there was evidence of significant associations between exposure to induced abortion and rates of mental health problems, the contribution of induced abortion to population rates of disorder was small. The estimated attributable fractions for the analyses summarised in Table 3 ranged from $1.5 \%$ to $5.5 \%$.

\section{Discussion}

In this paper we have used extensive data gathered over the course of a 30-year longitudinal study to examine the links between a series of pregnancy outcomes (abortion, pregnancy loss, unwanted pregnancy leading to live birth, and other live birth) and common mental health outcomes, including depression, anxiety, suicidal behaviours and substance use disorders. The major finding of this analysis is that even following extensive control for prospectively and concurrently measured confounders, women who had had abortions had rates of mental health problems that were about $30 \%$ higher than rates of disorder in other women. Although rates of all forms of disorder were higher in women exposed to abortion, the conditions most associated with abortion included anxiety disorders and substance use disorders. In contrast, none of the other pregnancy outcomes (pregnancy loss, live birth following unwanted pregnancy or a pregnancy having an initial adverse reaction, and other live birth) was consistently related to significantly increased risks of mental health problems.

Although exposure to abortion was associated with significant increases in risks of mental health problems, the overall effects of abortion on mental health proved to be small. Estimates of the attributable fraction suggested that exposure to abortion accounted for $1.5-5.5 \%$ of the overall rates of mental disorder in this cohort.

\section{Evidence of causality}

These findings are consistent with the view that exposure to abortion has a small causal effect on the mental health of women. The following lines of evidence support a causal conclusion.

(a) Consistency of associations: In this study data gathered at different ages and on different mental health outcomes showed generally consistent tendencies for women exposed to abortion to be at increased risks of mental health problems (online Table DS1).

(b) Resiliency to control for confounding: Associations between exposure to abortion and mental health proved to be resilient to extensive controls for confounding factors. Over 30 confounding variables were considered in the analyses reported. These variables included measures of childhood socio-economic background, family functioning, exposure to child abuse, individual factors, prior mental health disorders, and exposure to stressful life events in adulthood (including violence victimisation). This list of covariates appears to cover all of the major sources of confounding suggested in the literature. ${ }^{4,12,20}$

(c) Temporal sequence: Associations between abortion and mental health outcomes were present for lagged models in which exposure to abortion was assessed 3-5 years prior to the period during which mental health outcomes were assessed (Tables 2 and 3).

(d) Availability of comparison groups: A frequent criticism of research into abortion and mental health is that studies have failed to include appropriate comparison groups. ${ }^{43}$ In this study we have included results for three comparison groups: pregnancy loss, live birth following an adverse reaction to pregnancy, and other live birth. After extensive adjustment for confounding, abortion was the only pregnancy outcome that was associated with consistent increases in risks of mental health problems. The specificity of the association

Table 3 Adjusted risk ratios $(95 \% \mathrm{Cl})$ for the effect of abortion on number of mental health problems by alternative strategies for defining pregnancy history and alternative lag rules

\begin{tabular}{|lccc|} 
& & Pregnancy history, $\mathrm{RR}(95 \% \mathrm{Cl})$ \\
\cline { 2 - 4 } Lag rule & $\begin{array}{c}\text { Combined prospective and } \\
\text { retrospective data }\end{array}$ & Prospective data only & Retrospective data only \\
\hline Concurrent & $1.37(1.16-1.62)$ & $1.45(1.21-1.73)$ & $1.30(1.10-1.55)$ \\
\hline Lagged 5 years & $1.32(1.05-1.67)$ & $1.04-1.73)$ & $1.26(0.99-1.61)$ \\
\hline Lagged 4 years & $1.35(1.07-1.70)$ & $1.29(0.99-1.69)$ & $1.26(0.99-1.62)$ \\
\hline Lagged 3 years & $1.24(0.96-1.61)$ & $(0.93-1.64)$ \\
\hline
\end{tabular}


between abortion and increased mental health risks further reinforces the view that the association is causal.

(e) Robustness of results: The same general conclusions were reached using a series of models that varied in the basis for assessing pregnancy history (prospective, retrospective and combined) and the lag between exposure and outcome (Table 3).

(f) Theoretical plausibility of results: The finding of a small adjusted association between exposure to abortion and mental health is consistent with evidence which suggests that for a minority of women abortion is a highly stressful life event which evokes distress, guilt and other negative feelings that may last for many years. $3,6,8,13$

Although the weight of the evidence favours the view that abortion has a small causal effect on mental health problems, other explanations remain possible. In particular it could be suggested that the small association between abortion and mental health found in this study could be explained by uncontrolled residual confounding. As in all naturalistic studies, control of nonobserved sources of confounding is difficult but not impossible and there are several ways in which better control of such confounding might be achieved. ${ }^{44,45}$ The most informative design in this area is likely to be provided by a discordant twin design in which the mental health of female monozygotic twin-pairs who are discordant for abortion exposure is studied. ${ }^{44}$ In addition, the study was not able to examine the role of abortion in more serious forms of mental illness.

\section{Implications}

The conclusions drawn above have important implications for the ongoing debates between pro-life and pro-choice advocates about the mental health effects of abortion. Specifically, the results do not support strong pro-life positions that claim that abortion has large and devastating effects on the mental health of women. ${ }^{46}$ Neither do the results support strong pro-choice positions that imply that abortion is without any mental health effects. ${ }^{47}$ In general, the results lead to a middle-of-the-road position that, for some women, abortion is likely to be a stressful and traumatic life event which places those exposed to it at modestly increased risk of a range of common mental health problems.

Finally, the findings of this study have some important implications for the legal status of abortion in societies such as New Zealand and the UK, where over $90 \%$ of abortions are authorised on the grounds that proceeding with the pregnancy would pose a serious threat to the woman's mental health. ${ }^{48,49}$ In general, there is no evidence in the literature on abortion and mental health that suggests that abortion reduces the mental health risks of unwanted or mistimed pregnancy. Although some studies have concluded that abortion has neutral effects on mental health, ${ }^{4,5,12,14}$ no study has reported that exposure to abortion reduces mental health risks. These trends are evident in the present study, which shows that although abortion was associated with increased risks of mental health problems, no increase was evident for those having unwanted pregnancies that came to term. Although these conclusions are limited by the relatively small number of unwanted pregnancies that came to term, there is nothing in this study that would suggest that the termination of pregnancy was associated with lower risks of mental health problems than birth following an unwanted pregnancy. This evidence clearly poses a challenge to the use of psychiatric reasons to justify abortion for women having unwanted pregnancies in jurisdictions that require evidence that pregnancy poses harm to the woman's health before termination of pregnancy can be authorised.

David M. Fergusson, PhD, L. John Horwood, MSC, Joseph M. Boden, PhD,

Christchurch Health and Development Study, University of Otago, Christchurch School of Medicine and Health Sciences, New Zealand

Correspondence: Professor David M. Fergusson, Christchurch Health nd Development Study, University of Otago, Christchurch School of Medicine and Health Sciences, PO Box 4345, Christchurch, New Zealand. Email: dm.fergusson@otago.ac.nz

First received 23 Jun 2008, final revision 11 Aug 2008, accepted 14 Aug 2008

\section{Acknowledgements}

This research was funded by grants from the Health Research Council of New Zealand, the National Child Health Research Foundation, the Canterbury Medical Research Foundation and the New Zealand Lottery Grants Board.

\section{References}

1 Major B, Cozzarelli C, Cooper ML, Zubek J, Richards C, Wilhite M, Gramzow RH. Psychological responses of women after first-trimester abortion. Arch Gen Psychiatry 2000; 57: 777-84.

2 Quinton WJ, Major B, Richards C. Adolescents and adjustment to abortion: are minors at greater risk? Psychol Public Policy Law 2001; 7: 491-514.

3 Reardon DC, Cougle JR. Depression and unintended pregnancy in the National Longitudinal Survey of Youth: a cohort study. BMJ 2002; 324: 151-2.

4 Schmiege S, Russo NF. Depression and unwanted first pregnancy: longitudinal cohort study. BMJ 2005; 331: 1303.

5 Taft AJ, Watson LF. Depression and termination of pregnancy (induced abortion) in a national cohort of young Australian women: the confounding effect of women's experience of violence. BMC Public Health 2008; 8: 75.

6 Broen AN, Moum T, Bodtker AS, Ekeberg O. The course of mental health after miscarriage and induced abortion: a longitudinal, five-year follow-up study. BMC Med 2005; 3: 18.

7 Cougle JR, Reardon DC, Coleman PK. Depression associated with abortion and childbirth: a long-term analysis of the NLSY cohort. Med Sci Monit 2003; 9: CR105-12.

8 Fergusson DM, Horwood LJ, Ridder E. Abortion in young women and subsequent mental health. J Child Psychol Psychiatry 2006; 47: 16-24.

9 Rees DI, Sabia JJ. The relationship between abortion and depression: new evidence from the fragile families and child wellbeing study. Med Sci Monit 2007; 13: CR430-6.

10 Pedersen W. Abortion and depression. A population-based longitudinal study of young women. Scand J Public Health 2008; 36: 424-8.

11 Cougle JR, Reardon DC, Coleman PK. Generalized anxiety following unintended pregnancies resolved through childbirth and abortion: a cohort study of the 1995 National Survey of Family Growth. J Anxiety Disord. 2005; 19: $137-42$.

12 Steinberg JR, Russo NF. Abortion and anxiety. What's the relationship? SOC Sci Med. 2008; 67: 238-52.

13 Pedersen W. Childbirth, abortion and subsequent substance use in young women: a population-based longitudinal study. Addiction 2007; 102: 1971-8.

14 Gilchrist AC, Hannaford PC, Frank P, Kay CR. Termination of pregnancy and psychiatric morbidity. Br J Psychiatry 1995; 167: 243-8.

15 Fergusson DM. Abortion and mental health. Psychiatr Bull 2008; 32: 321-4.

16 Royal College of Psychiatrists. Position statement on women's mental health in relation to induced abortion. 14 March 2008. Royal College of Psychiatrists, 2008 (http://www.rcpsych.ac.uk/members/currentissues/ mentalhealthandabortion.aspx).

17 Major B. Psychological implications of abortion - highly charged and rife with misleading research. CMAJ 2003; 168: 1257-8

18 Morris $K$, Orr $F$. Is there a role for psychiatry in late termination of pregnancy? Aust N Z J Psychiatry 2007; 41: 709-17.

19 CMAJ. Unwanted results: the ethics of controversial research. CMAJ 2003; 169: 93.

20 Adler NE, David HP, Major BN, Roth SH, Russo NF, Wyatt GE. Psychological responses after abortion. Science 1990; 248: 41-4.

21 Reardon DC, Cougle JR, Rue VM, Shuping MW, Coleman PK, Ney PG. Psychiatric admissions of low-income women following abortion and childbirth. CMAJ 2003; 168: 1253-6. 
22 Cohen SA. Abortion and mental health: myths and realities. Guttmacher Rep Policy Review 2006; 9: 8-12.

23 Costello A, Edelbrock C, Kalas R, Kessler M, Klaric SA. Diagnostic Interview Schedule for Children (DISC). National Institute of Mental Health, 1982.

24 World Health Organization. Composite International Diagnostic Interview (CIDI). WHO, 1993.

25 American Psychiatric Association. Diagnostic and Statistical Manual of Mental Disorders (4th edn) (DSM-IV). APA, 1994.

26 Elley WB, Irving JC. Revised socio-economic index for New Zealand. NZJES 1976; 11: 25-36.

27 Straus MA. Measuring intrafamily conflict and violence: the Conflict Tactics (CT) scales. J Marriage Fam 1979; 41: 75-88.

28 Fergusson DM, Horwood LJ. Exposure to interparental violence in childhood and psychosocial adjustment in young adulthood. Child Abuse Negl 1998; 22 339-57.

29 Armsden GC, Greenberg MT. The inventory of parent and peer attachment. Individual differences and their relationship to psychological well-being in adolescence. I Youth Adolesc 1987; 16: 427-54.

30 Fergusson DM, Lynskey MT, Horwood LJ. Childhood sexual abuse and psychiatric disorder in young adulthood: I. Prevalence of sexual abuse and factors associated with sexual abuse. J Am Acad Child Psychiatry 1996; 35 : 1355-64.

31 Fergusson DM, Lynskey MT. Physical punishment/maltreatment during childhood and adjustment in young adulthood. Child Abuse Negl 1997; 21: 617-30

32 Eysenck HM, Eysenck SBG. Manual of the Eysenck Personality Inventory. London University Press, 1964.

33 Coopersmith S. Self-esteem Inventory (SEI). Consulting Psychologists Press, 1981.

34 Cloninger CR. A systematic method for clinical description and classification of personality variants. A proposal. Arch Gen Psychiatry 1987; 44: 573-88.

35 Rutter M, Tizard J, Whitmore K. Education, Health and Behaviour. Longmans, 1970.
36 Conners CK. A teacher rating scale for use in drug studies with children. Am J Psychiatry 1969; 126: 884-8.

37 Conners CK. Symptom patterns in hyperkinetic, neurotic and normal children. Child Dev 1970; 41: 667-82.

38 Wechsler D. Manual for the Wechsler Intelligence scale for Children Revised. Psychological Corporation, 1974.

39 American Psychiatric Association. Diagnostic and Statistical Manual of Mental Disorders (3rd edn, revised) (DSM-III-R). APA, 1987.

40 Lewis CE, Seigel JM, Lewis MA. Feeling bad: exploring sources of distress among pre-adolescent children. Am J Public Health 1984; 74: 117-22.

41 Bruzzi P, Green SB, Byar DP, Brinton LA, Schairer C. Estimating the population attributable risk for multiple risk factors using case-control data. Am J Epidemiol 1985; 122: 904-14.

42 Carlin JB, Wolfe R, Coffey C, Patton GC. Tutorial in Biostatistics. Analysis of binary outcomes in longitudinal studies using weighted estimating equations and discrete-time survival methods: prevalence and incidence of smoking in an adolescent cohort. Stat Med 1999; 18: 2655-79.

43 Major B, Appelbaum M, Beckman L, Dutton MA, Russo NF, West C. Report of the APA Task Force on Mental Health and Abortion 8/13/2008. American Psychological Association, 2008 (http://www.apa.org/releases/abortionreport.pdf).

44 Cameron AC, Trivedi PK. Regression Analysis of Count Data. Cambridge University Press, 1998.

45 Kendler KS. Of genes and twins. Psychol Med 2003; 33: 763-8.

46 Agee MC. The health risks of abortion. Family Research Council, 2003 (http:// www. federalobserver.com/archive. php?aid=8193)

47 Planned Parenthood. Abortion. Planned Parenthood Federation of America, 2006 (http://www.plannedparenthood.org/health-topics/abortion-4260.htm)

48 Statistics New Zealand. Abortions: Year Ended December 2007 (http://www.stats.govt.nz/NR/rdonlyres/49CD5979-F29B-4C1F-9B889F610FBDEA2D/0/abortionsyearendeddec07hotp.pdf).

49 Government Statistical Service. Abortion Statistics, England and Wales: 2003. Department of Health, 2004. 